\title{
Philosophiques
}

\section{Les énoncés de croyance et l'énigme de Kripke}

\section{Michel Seymour}

Volume 15, numéro 1, printemps 1988

URI : https://id.erudit.org/iderudit/027031ar

DOI : https://doi.org/10.7202/027031ar

Aller au sommaire du numéro

Éditeur(s)

Société de philosophie du Québec

ISSN

0316-2923 (imprimé)

1492-1391 (numérique)

Découvrir la revue

Citer cet article

Seymour, M. (1988). Les énoncés de croyance et l'énigme de Kripke. Philosophiques, 15(1), 5-29. https://doi.org/10.7202/027031ar

\section{Résumé de l'article}

Je propose une solution à l'énigme de Kripke en distinguant tout d'abord les usages matériel et intentionnel du verbe " croire ». L’usage intentionnel est ensuite analysé à partir de l'usage matériel. Une croyance intentionnelle n'est rien d'autre qu'une croyance matérielle réitérée, c'est-à-dire, une croyance matérielle que l'agent entretient au sujet de ses propres croyances. L'énigme de Kripke peut alors être résolue pourvu que l'on accepte aussi l'inscriptionnalisme. On est ainsi en mesure de reconnaître que Pierre a bel et bien des " croyances » contradictoires, mais seulement au sens matériel de l'expression. Il n'a cependant pas de croyances intentionnelles contradictoires puisque les contenus de croyance sont des inscriptions et qu'il se croit luimême en rapport avec ces inscriptions. 


\title{
LES ÉNONCÉS DE CROYANCE ET L'ÉNIGME DE KRIPKE ${ }^{1}$
}

\author{
par Michel Seymour
}

\begin{abstract}
RÉSUMÉ. Je propose une solution à l'énigme de Kripke en distinguant tout d'abord les usages matériel et intentionnel du verbe «croire ». L'usage intentionnel est ensuite analysé à partir de l'usage matériel. Une croyance intentionnelle n'est rien d'autre qu'une croyance matérielle réitérée, c'est-à-dire, une croyance matérielle que l'agent entretient au sujet de ses propres croyances. L'énigme de Kripke peut alors être résolue pourvu que l'on accepte aussi l'inscriptionnalisme. On est ainsi en mesure de reconnaittre que Pierre a bel et bien des «croyances » contradictoires, mais seulement au sens matériel de l'expression. Il n'a cependant pas de croyances intentionnelles contradictoires puisque les contenus de croyance sont des inscriptions et qu'il se croit luimême en rapport avec ces inscriptions.
\end{abstract}

ABSTRACT. I offer a solution to Kripke's puzzle by first distinguishing between material and intentional uses of "believe". The intentional use is then analysed in terms of material uses. An intentional belief is nothing but a material belief that an agent entertains towards his own beliefs. Kripke's puzzle can then be solved if inscriptionalism is also accepted. It can indeed be claimed that Pierre has contradictory "beliefs", but only in the material sense of the word. However, he does not have intentional contradictory beliefs since the contents of his beliefs are inscriptions and he represents himself as being directed towards those inscriptions.

Je me propose dans les pages qui suivent de présenter l'énigme de Kripke portant sur les énoncés de croyance et de faire

1. Ce texte est la version remaniée et augmentée d'une communication présentée à l'ACFAS en mai 86 à l'occasion d'une table ronde portant sur l'énigme de Kripke. Le texte de référence est $\mathrm{S}$. KRIPKE “A puzzle about belief », dans A. MARgalit, (ed.) Meaning and Use, D. Reidel pub. co., 1979, pp. 239-283. Avant d'arriver à cetre dernière version, j'aurai bénéficié des commentaires de $\mathrm{K}$. Arnold, M. Lagueux, D. Laurier, F. Lepage, J. Leroux, C. Panaccio et C. Travis. 
ensuite un certain nombre de remarques sur des points que je juge essentiels à sa résolution. Je crois que Kripke a raison de penser qu'il y a bel et bien une énigme (c'est d'ailleurs là sa thèse principale) et c'est pourquoi les éléments de réponse que j'apporte à cette question veulent permettre une solution et non une dissolution de l'énigme. Je voudrais cependant d'abord et avant tout replacer la discussion dans un contexte plus large et dire quelques mots sur le débat que Kripke entretient avec les philosophies sémantiques d'allégeance frégéenne puisque c'est de là qu'origine la discussion dans laquelle il s'engage dans son article.

\section{I}

Déjà à l'époque de Naming and Necessity ${ }^{2}$, Kripke s'en prend aux théories sémantiques qui procèdent d'une distinction entre le sens et la référence ou entre l'intension et l'extension des expressions. En ce qui a trait au problème de la référence des noms propres, elles prennent souvent la forme de théories «descriptivistes», c'est-à-dire de théories qui supposent que les noms propres expriment un sens susceptible d'être rendu par une ou des descriptions définies et ont comme référence l'unique entité qui exemplifie le(s) concept(s) exprimé(s) par la (les) description(s). Il faudrait bien prendre garde de ne pas trop rapidement confondre cette théorie avec la théorie russellienne qui suppose seulement que les noms propres sont des abréviations pour des descriptions. Russell n'invoque pas une distinction entre le sens et la référence des expressions; bien au contraire, il s'y oppose explicitement, dans «On Denoting » notamment ${ }^{3}$. Kripke, lui-même, a reconnu l'importance d'une telle nuance ${ }^{4}$.

Quoi qu'il en soit, il s'en prend d'abord et avant tout à la distinction frégéenne et il importe de comprendre pourquoi. On peut présumer qu'une motivation essentielle provient de ce que l'on veuille garantir le caractère public des significations et ne pas céder au psychologisme. Il faut reconnaître que le psychologisme

2. S. KRIPKE, Naming and Necessity, Basil Blackwell, 1980. L'ouvrage est paru aussi dans D. Davidson, G. Harman, (eds) Semantics of Natural Language, D. Reidel, 1972, 253-355.

3. B. Russell, «On Denoting», Mind, 1905.

4. Naming and Necessity, p. 27, note 4; "A puzzle about belief», p. 271. 
paraît pratiquement inévitable lorsqu'on tente d'appliquer la distinction frégéenne aux langues naturelles, car le «sens » y varie d'un locuteur à l'autre. Même en supposant que les significations subjectives des locuteurs soient au départ susceptibles d'être communiquées, leur caractère variable fait en sorte qu'ultimement la frontière entre sémantique et pragmatique se brouille et cette difficulté n'a jamais vraiment été surmontée par les philosophes frégéens. Kripke a sans doute aussi comme autre motivation la défense du réalisme aristotélicien, mais cette défense est davantage chez lui une conséquence de la face positive de sa théorie de la référence. Si l'on s'en tient à sa face négative et donc à sa critique de la tradition frégéenne, c'est l'anti-psychologisme qui s'avère être la motivation essentielle.

Il faut se rappeler que dans son célèbre article, Frege invoque différentes raisons pour justifier la distinction entre le sens et la référence des expressions's. Le cas des énoncés d'identité est traditionnellement considéré, mais aussi celui des contextes obliques et, en particulier, celui des énoncés d'attitudes propositionnelles. Les expressions qui entrent sous la portée des verbes psychologiques peuvent difficilement, selon Frege, avoir pour seule fonction sémantique celle de référer aux objets. Si tel était leur seul rôle sémantique, les expressions co-désignatives pourraient alors dans ces contextes être substituées les unes aux autres sans que la valeur de vérité de la proposition entière ne soit affectée. Autrement dit, on devrait être en mesure d'appliquer dans de tels contextes le principe de substitution des identiques. Or le fait est, justement, qu'un locuteur peut croire que Phosphorus est une planète sans croire que Hesperus est une planète, et ce, même si les deux noms réfèrent à une même entité, soit Vénus. La solution frégéenne consiste à supposer que les expressions désignent en contexte épistémique leur sens ordinaire et non leur référence ordinaire.

Kripke s'oppose à cette solution et cherche donc à porter un diagnostic différent. Les contextes d'attitudes propositionnelles sont traditionnellement déclarés « opaques » et le critère essentiel d'opacité référentielle est l'échec du principe de substitution des

5. G. FrEGE, «Sens et Dénotation», dans Écrits Logiques et Philosophiques, Éd. du Seuil, Paris, 1971. 
identiques. Une fois que l'opacité référentielle est reconnue, il n'y a qu'un pas à faire pour conclure que les expressions doivent bien avoir comme valeur sémantique autre chose que leur référence ordinaire. L'énigme que Kripke considère ne fait cependant aucunement intervenir le principe de substitution des identiques ${ }^{6}$. L'opacité référentielle n'est alors plus le phénomène qu'il faut chercher à expliquer et, par voie de conséquence, l'erreur ne semble plus être de supposer que les expressions n'ont que leur référence ordinaire comme valeur sémantique. Le problème est ailleurs. Kripke ne nous dit pas où, il se contente de nous montrer que l'énigme est véritable et qu'elle intervient quelle que soit la théorie des noms propres adoptée. Si cela était admis, une motivation essentielle en faveur de la théorie descriptiviste disparaîtrait et c'est là ce que Kripke cherche à montrer d'abord et avant tout dans son article.

\section{II}

Kripke commence par introduire deux principes qui semblent ne pas prêter à la controverse. L'admission de ces principes suffit cependant à générer une énigme. Le premier, le principe de "décitation» ( « disquotational principle »), stipule que l'assentiment d'un locuteur sémantiquement compétent à un énoncé « $\mathrm{p}$ » est une condition suffisante pour qu'il croit que $\mathrm{p}^{7}$. Il ne s'agit pas, au départ, de prétendre que la croyance se réduit à une relation entre un locuteur et un énoncé. On suppose seulement que l'assentiment à une phrase « $\mathrm{p}$ » peut impliquer la croyance que $\mathrm{p}$ pourvu que certaines conditions soient satisfaites. Au moins deux conditions doivent être introduites. Tout d'abord, on impose une condition de sincérité pour éliminer d'emblée les cas où le locuteur donne son assentiment dans le contexte d'une pièce de théâtre ou d'un propos ironique. On impose ensuite une condition de réflexivité du locuteur pour éliminer les cas d'inattention. Cela revient à supposer, entre autres choses, que le locuteur est suffisamment perspicace pour être en mesure de déceler une contradiction logique dans une formule, quel que soit son degré de complexité.

6. «A puzzle about belief », p. 253.

7. “A puzzle about belief», pp. 248-49. 
Le deuxième principe admis par Kripke est le principe de traduction et il stipule que si un énoncé " $\mathrm{p}$ " " est une bonne traduction de " $p$ », alors il a la même valeur de vérité que « $p{ }^{8}$. Ce principe est admis bien qu'il soit falsifié dans certains cas exceptionnels comme, par exemple, lorsqu'on rapporte en français les propos du président Reagan. La traduction adéquate de «Reagan said "God bless America" " est «Reagan a dit "Que Dieu bénisse l'Amérique" » et elle transforme un énoncé vrai (par hypothèse) en un énoncé faux puisque Reagan ne parle pas français. Le principe peut quand même être admis pourvu que l'on ne fasse pas appel à de tels exemples problématiques.

Voici donc l'énigme. Pierre est un locuteur du français. Il entend parler de Londres comme d'une très jolie ville et il s'accorde à dire que Londres est jolie. Puisqu'il est sincère et réflexif en disant cela, on peut en conclure, par le principe de décitation, qu'il croit que Londres est jolie. Il se rend plusieurs mois plus tard en Angleterre et, tout en préservant ses croyances antérieures, finit par s'installer dans un quartier pauvre d'une certaine ville où les gens ne sont pas très éduqués. Il apprend l'anglais directement au contact des gens plutôt que par un manuel de traduction. On lui indique que la ville dans laquelle il se trouve est «London». Après quelques mois exaspérants, il finit par conclure : London is not pretty. En vertu du principe de décitation, on peut inférer en anglais: Pierre believes that London is not pretty. Si cet énoncé est vrai, on peut en outre conclure, en invoquant le principe de traduction, que la traduction française est vraie elle aussi, à savoir, «Pierre croit que Londres n’est pas jolie». Mais alors, Pierre croit-il que Londres est jolie ou que Londres n'est pas jolie? Ou a-t-il les deux croyances? Il faut remarquer qu'on est arrivé à de telles croyances contradictoires sans utiliser le principe de substitution des identiques.

Au surplus, en admettant une version forte du principe de décitation selon laquelle refuser son assentiment à un énoncé « $\mathrm{p}$ » entraîne la non-croyance que $p$, une conséquence néfaste s'ensuit, non plus seulement pour Pierre, mais pour nous aussi. Si Pierre est réflexif, il ne peut donner son assentiment à «London is not

8. «A puzzle about belief», p. 250. 
pretty et «London is pretty» en même temps. Puisque, par hypothèse, il affirme le premier, il refusera d'accorder son assentiment au deuxième et si la version forte du principe de décitation est admise, la conclusion suivante peut être tirée en anglais : Pierre does not believe that London is pretty. En vertu du principe de traduction, on a que Pierre ne croit pas que Londres est jolie. Mais alors Pierre croit-il ou ne croit-il pas que Londres est jolie? Ce n'est plus seulement Pierre qui est en cause ici car nous sommes nous-mêmes confrontés à une contradiction dans nos attributions d'attitudes psychologiques à Pierre.

\section{III}

L'énigme que Kripke nous demande de considérer a trait aux énoncés de croyance et elle se pose au cas où ils sont utilisés sur le mode de dicto 9 . Aussi, avant d'aller plus loin, il convient de dire quelques mots sur la distinction de re/de dicto. En général, on dira qu'une croyance est de dicto si elle se rapporte à un énoncé ou à la proposition qu'il exprime et on dira qu'elle est de re si elle se rapporte plutôt à l'objet dont parle l'énoncé ou la proposition qu'il exprime. Une distinction analogue peut être faite pour les modalités logiques. Un énoncé comme «Il est nécessaire que $\mathrm{F}(\mathrm{A})$ » peut recevoir au moins deux lectures, l'une de dicto l'autre de re. Dans le premier cas, il est dit que l'énoncé « $F(A)$ », ou la proposition qu'il exprime, est vrai dans tous les mondes possibles. Dans le second cas, on affirme plutôt que l'objet $A$ a comme propriété essentielle d'être $\mathrm{F}$, ou est $\mathrm{F}$ dans tous les mondes possibles. Selon que la première ou la deuxième lecture est adoptée, l'énoncé modal se rapporte à un énoncé (une proposition) ou à l'objet dont parle l'énoncé (la proposition).

Kripke ne veut considérer que les usages de dicto des énoncés de croyance et il peut sembler qu'il en fasse une condition nécessaire pour que l'énigme apparaisse. À supposer cependant que les noms soient sans connotation, il faudrait alors reconnaître que les lectures de dicto et de re sont équivalentes et qu'il n'y a plus de distinction importante à établir entre elles. En effet, il n'y a pas de véritable distinction entre une croyance qui se rapporte à un objet

9. «A puzzle about belief », p. 242. 
et une croyance qui se rapporte à une proposition lorsqu'il s'agit d'une proposition singulière, et les énoncés qui contiennent des termes singuliers non-connotatifs déterminent des propositions singulières. Si la théorie millienne des noms propres est adéquate, l'énigme de Kripke ne peut être formulée strictement en termes de dicto.

Tout dépend en fait ici de la terminologie adoptée. En souscrivant aux définitions proposées plus haut, on reste relativement indifférent au débat entre descriptivistes et causalistes en théorie de la référence. Selon notre terminologie, les énoncés d'attitudes ont autant une lecture de dicto que de re même lorsque les termes singuliers sont jugés non connotatifs et que les énoncés dans lesquels ils entrent déterminent des propositions singulières. Une lecture de dicto est compatible avec la théorie millienne de la référence et Kripke ne va pas à l'encontre de sa propre théorie en voulant formuler son énigme en termes de dicto.

La question demeure cependant de savoir pourquoi Kripke cherche à formuler de cette façon l'énigme, surtout si la frontière entre le de dicto et le de re est à ce point ténue. La raison est très simple. L'énigme consiste en ce que Pierre est confronté à une contradiction logique, c'est-à-dire qu'on est progressivement amené à reconnaître, en un certain sens, que Pierre croit que pet $-p$, et ce, bien qu'il soit par hypothèse un logicien perspicace. Si les énoncés sont interprétés seulement sur le mode de re, Pierre ne croit plus des propositions contradictoires et dirige plutôt ses états intentionnels sur des états de choses métaphysiquement incompatibles ${ }^{10}$. C'est un fait métaphysique et non logique qui explique pourquoi la ville de Londres, elle-même, ne peut être à la fois jolie et pas jolie. Les croyances de Pierre ne sont logiquement contradictoires que si elles sont de dicto.

Il ne faut pas en cela comprendre cependant que l'énigme de Kripke ne se poserait plus si les énoncés d'attitudes étaient interprétés sur le mode de re car, en admettant la version forte du principe de décitation, on arrive à la conclusion que celui qui rapporte la croyance de Pierre est confronté à une contradiction logique. L'énigme persiste sous une certaine forme même après

10. Cetre idée est due à Daniel Laurier. 
que l'on se soit exclusivement rabattu sur des lectures de re des croyances de Pierre parce que le rapporteur arrive à la conclusion suivante au sujet de Londres : Pierre la croit et ne la croit pas jolie.

Il faut aussi remarquer que l'énigme se pose pour une théorie millienne de la référence même en admettant le principe de décitation dans sa version affaiblie lorsque le contenu des attitudes est assimilé à des inscriptions. Selon cette hypothèse, en effet, même si les énoncés déterminent des propositions singulières et, par la même occasion, des états de choses métaphysiquement incompatibles, Pierre, lui, est confronté à des énoncés logiquement contradictoires. Ou du moins est-ce là la conclusion qu'on semble forcé de tirer bien que l'on ait postulé au départ une capacité réflexive chez Pierre.

Est-ce à dire cependant que, sans l'inscriptionnalisme, l'énigme ne tient qu'à l'admission du principe de décitation dans sa version forte pour les théoriciens de la référence directe? Si les énoncés déterminent des propositions singulières, n'y a-t-il plus de contradiction logique chez Pierre? Dans ce cas, il semble qu'il faille plutôt dire que Pierre est simultanément confronté à une contradiction logique et à des états de choses métaphysiquement incompatibles. Là encore, l'énigme n'est pas totalement dissoute. Pour vraiment parvenir à la faire disparaître, le philosophe millien devrait en outre ajouter que Pierre peut ignorer la proposition qu'il croit, mais ce serait là déjà être en train d'apporter une solution plutôt que de prétendre qu'il n'y a pas d'énigme.

Donc si Pierre peut ignorer le contenu de sa croyance (et un argument possible ici serait que Pierre peut utiliser de manière compétente le terme «Londres» sans savoir pour autant quel objet est le référent du terme), si le contenu de la croyance est une proposition, si les termes singuliers sont non connotatifs et les énoncés dans lesquels ils apparaissent déterminent des propositions singulières, alors Pierre n'est plus lui-même confronté à une contradiction logique et l'énigme disparaît. Mais comme on vient de le faire remarquer, une hypothèse nouvelle est apparue, celle selon laquelle Pierre pourrait croire des propositions qu'il n'appréhende pas vraiment intentionnellement, de telle sorte que l'on ne peut prétendre qu'il n'y a pas d'énigme, et ce, même lorsqu'on s'en tient à la version faible du principe de décitation. On fait aussi 
l'hypothèse que les objets des attitudes sont des propositions et, là encore, il s'agit d'un élément de solution et non de dissolution. Quoi qu'il en soit, l'énigme persiste dès que l'on admet la version forte du principe et ce dernier point est décisif car, comme on le verra plus loin, une interprétation particulière rend ce principe tout à fait acceptable.

Si les théoriciens de la référence directe sont de toute façon confrontés à une énigme, il convient alors, pour Kripke, de la formuler en des termes qui soient intelligibles aux diverses parties concernées. Il veut bien montrer que l'énigme se pose aussi aux philosophes qui traitent les noms comme connotatifs, ce qui lui permet de critiquer ceux qui s'en prennent au point de vue millien. Il faut donc chercher d'abord et avant tout à formuler l'énigme en ne considérant que le cas où les croyances de Pierre sont interprétées sur le mode de dicto. Ce faisant, on se place sur un terrain où les deux parties peuvent s'entendre. Les frégéens ne reconnaissent en effet que les croyances de dicto alors que les milliens admettent que certaines croyances peuvent être interprétées autant sur le mode de re que de dicto. En restant sur le mode de dicto, on ne tranche pas en faveur d'une théorie particulière de la référence et on reste à un niveau de généralité acceptable aux deux parties.

Une fois qu'on s'est placé ainsi sur le terrain de l'adversaire, on peut montrer que la distinction entre le sens et la référence, à laquelle les frégéens ont traditionnellement recours pour bloquer les inférences indésirées, est sans utilité ici. Cette distinction est inutile dans le cas qui nous occupe étant donné que, par hypothèse, les expressions «Londres » et «London» sont jugées de bonnes traductions l'une pour l'autre.

\section{IV}

La solution que je voudrais mettre de l'avant consiste à distinguer tout d'abord les usages matériel et intentionnel des verbes psychologiques. Quand on rapporte un état psychologique sur le mode matériel, on met l'agent en relation avec un contenu qu'il n'appréhende pas nécessairement. C'est un peu comme si on lui passait en quelque sorte par-dessus la tête pour lui attribuer un contenu qui peut lui échapper. Dans un usage intentionnel du 
verbe psychologique, par contre, on rapporte l'état psychologique en faisant intervenir un contenu dont on suppose qu'il est appréhendé par l'agent.

On dira, par exemple, en accord parfait avec le sens littéral du verbe psychologique, qu'Edipe voulait marier sa mère même si ses états intentionnels ne concernaient que Jocaste. Il y a un sens littéral du verbe selon lequel l'énoncé qui attribue à $\mathrm{CEdipe} \mathrm{le} \mathrm{désir}$ de marier sa mère est un énoncé vrai. De la même manière, si un locuteur croit que p et que $p$ implique $q$, alors, en un certain sens, il croit aussi que q. Cet usage du verbe "croire», qui s'accorde parfaitement avec notre intuition de locuteur, est encore une fois l'usage matériel. Comme dernier exemple, on pourrait rapporter l'assentiment de Reagan à l'effet que les Américains sont les plus forts comme étant, au fond, une relation particulière de croyance que le président entretient avec la phrase « les Américains sont les plus forts ». Bien sûr, le président n'a pas d'état intentionnel de croyance qui le relie à une phrase du français, pas plus qu'un agent n'a de croyance intentionnelle dirigée sur l'ensemble des conséquences logiques de ses croyances, ou qu'CEdipe n'éprouve de désir intentionnel de marier sa mère. Il y a quand même des usages non intentionnels des verbes psychologiques et, si l'on s'accorde à dire que ces usages sont tout à fait littéraux, il nous faut reconnaître alors qu'une ambiguité fondamentale affecte ces verbes. Ils peuvent être utilisés, tantôt sur le mode intentionnel, pour reproduire le contenu " appréhendé » par le locuteur, tantôt sur le mode matériel, pour mettre les états intentionnels du locuteur en relation avec des contenus qui peuvent les transcender.

Avant d'aller plus loin, $\mathrm{j}$ 'aimerais faire quelques précisions sur la distinction que je viens d'introduire. Il ne faudrait pas la confondre avec une autre, à savoir, la distinction entre le mode de dicto et le mode de re. Habituellement, la distinction entre croyance de dicto et croyance de re qualifie deux sortes de modalités épistémiques, deux sortes d'attitudes possédées par le locuteur. Le locuteur peut être en rapport avec un certain contenu ou avec un objet « lui-même ». Cependant, notre distinction concerne non pas deux sortes d'attitudes, mais plutôt deux façons de les rapporter. En effet, l'agent n'a en réalité que des états psychologiques intentionnels. Toutes ses croyances sont, pour ainsi dire, des croyances qu'il s'attribuerait lui-même, si l'on fait exception des 
croyances inconscientes pour les fins de la discussion. Il importe de noter que les croyances qui lui sont attribuées sur le mode matériel n'ajoutent rien à l'ensemble de ses croyances effectives et ne font que mettre en rapport ces dernières avec des contenus autres que ceux qu'il considère effectivement. Une attribution de croyance matérielle se fait sans tenir compte de l'intentionnalité de l'agent alors que c'est précisément ce dernier fait qui motive une attribution de croyance intentionnelle.

Supposons cependant que, comme Searle, l'on veuille réduire la distinction de re/de dicto à une distinction entre deux sortes de rapports ${ }^{11}$. En ce cas, il pourrait sembler que l'usage de dicto coïncide avec l'usage intentionnel alors que l'usage de re coïncide avec l'usage matériel, mais il n'en est rien. Un rapport de re de croyance n'est alors quand même qu'une espèce particulière de rapport matériel. Un rapport de croyance fait sur le mode matériel peut mettre l'agent en relation avec un énoncé dans une autre langue ou avec une conséquence logique de ses croyances, ainsi qu'on l'a indiqué plus haut, et cela montre que l'usage matériel s'accorde autant avec une attribution de dicto qu'avec une attribution de re. Par ailleurs, la véritable question au sujet des croyances de re concerne le cas des croyances intentionnelles. On trouvera peutêtre absurde de supposer des croyances intentionnelles de re, mais c'est là une question de fait et non une question conceptuelle. Searle ne veut pas admettre des croyances intentionnelles de re et c'est pourquoi il conçoit la distinction de re/de dicto comme s'appliquant seulement au niveau des rapports. Je partage en partie le scepticisme de Searle, mais je m'objecte néanmoins à sa réduction de la distinction de re/de dicto à une distinction entre deux sortes de rapports faits par un tiers.

La deuxième mise au point que je veux faire est la suivante. Il ne faut pas confondre l'usage matériel des verbes psychologiques avec l'attribution d'une relation que le locuteur entretiendrait avec des états de choses existants. L'usage matériel n'implique pas que le contenu attribué soit un état de choses existant. Pierre peut croire matériellement que Londres n'est pas jolie même si, de fait,

11. J. SEARLE, «Intentionality and the use of language » dans A. MARGalit (ed.), Meaning and $U_{s e}, \mathrm{D}$. Reidel pub. co., 1979, 181-197. 
Londres est jolie. Inversement, une attribution intentionnelle est compatible avec le fait que l'état de choses correspondant existe.

Enfin, la distinction introduite n'implique encore rien quant à la nature des contenus de croyance. Les contenus de croyance peuvent être aussi bien des entités intentionnelles que des situations (des états de choses possibles) ou des inscriptions.

Les deux sortes de rapports que j'ai introduits ne sont toutefois pas irréductibles l'un à l'autre. En réalité, l'usage intentionnel du verbe, au sens où je l'entends ici, peut s'analyser à partir de l'usage matériel. Celui qui fait un rapport intentionnel essaie de se conformer à la représentation que se fait l'agent de ses propres états psychologiques. Quand l'attribution est intentionnelle, on cherche à reproduire le contenu de croyance que l'agent s'attribuerait lui-même. Cette façon de voir les choses suggère une analyse très simple du rapport intentionnel de croyance. Ce rapport n'est rien d'autre qu'un rapport matériel qui met l'agent en relation avec ses propres croyances. Dire que Pierre croit intentionnellement que $p$, c'est dire que Pierre se représente comme ayant la croyance que $p$. Une croyance intentionnelle n'est alors rien d'autre qu'une espèce particulière de croyance matérielle, celle qu'un agent entretient au sujet de sa propre croyance :
A croit matériellement $[p]$ et
A croit intentionnellement que $\mathrm{p}=\mathrm{df} \quad \mathrm{A}$ croit matériellement que
A croit matériellement $[\mathrm{p}]$.

J'ai volontairement employé les crochets pour ne pas m'engager, pour le moment, à une caractérisation particulière des contenus de croyance.

\section{V}

La distinction entre les deux usages des verbes psychologiques et l'analyse de l'usage intentionnel en un usage matériel réitéré constituent presque l'essentiel de ce dont on a besoin pour résoudre l'énigme de Kripke. Mais la distinction introduite ne présuppose pas la vérité d'une quelconque caractérisation des contenus de croyance. Ces contenus peuvent aussi bien être des entités intentionnelles, des situations ou des inscriptions. Je voudrais cependant endosser une version particulière de l'inscriptionnalisme. L'adoption 
d'une théorie inscriptionnaliste s'avère être en fait une étape essentielle qui permet d'arriver à une solution de l'énigme.

D'une manière générale, un énoncé de croyance matérielle comme «A croit que $s$ », où « $s$ » est une phrase ne contenant pas d'expressions déictiques, est de la forme logique suivante:

$(\Sigma \mathrm{p})((\mathrm{A}$ croit $\ll \mathrm{p} »)-(\mathrm{A}$ croit $« \mathrm{p} » \equiv \mathrm{A}$ croit $\ll \mathrm{s}\rangle))$.

Dans cette formule, le quantificateur particulier « $\Sigma$ » est substitutionnel et il lie la variable « $\mathrm{p}$ » à laquelle correspond une classe de substitution contenant comme éléments des énoncés d'un ordre déterminé. La variable apparaît dans un contexte de citation mais il ne faut pas oublier que la quantification substitutionnelle à l'intérieur d'un tel contexte n'est pas problématique comme elle l'est généralement lorsque le quantificateur est objectuel. Le fait de mettre la variable entre guillemets n'a pas non plus pour effet d'introduire un nom de variable lorsque celle-ci est substitutionnelle. Les guillemets sont plutôt une sorte de fonction descriptive qui prend comme « argument » dans ce cas un énoncé appartenant à la classe de substitution et qui a ce même énoncé comme "valeur». Ils peuvent être comparés à la fonction descriptive «l'individu identique à y » qui a elle aussi comme valeur l'entité qu'elle prend comme argument.

Le cas des énoncés d'attitudes contenant des déictiques est plus complexe. La subordonnée ne peut plus recevoir une traduction homophonique sans que la valeur de vérité de l'énoncé initial ne soit modifiée. Par exemple, l'énoncé :

Pierre croit que je viendrai demain

ne saurait être traduit par

$(\Sigma \mathrm{p})(($ Pierre croit $« \mathrm{p} ») \cdot($ Pierre croit $\ll \mathrm{P} » \equiv$ Pierre croit $\ll$ je viendrai demain ») ).

Le problème vient du fait que nos traductions se font dans des «formes logiques» et que les «formes logiques» spécifient la structure du contenu des énoncés. En général, il n'y a pas de problème à entreprendre sur le champ une spécification du contenu d'un énoncé lorsque celui-ci ne contient pas de déictiques et qu'il a donc un caractère stable. Pour les énoncés qui contiennent des déictiques, il faut commencer par déterminer le contenu de l'énoncé 
relativement au contexte, à la suite de quoi l'énoncé reçoit l'interprétation habituelle. Ainsi, l'énoncé qu'on considère peut, relativement à un contexte d'énonciation, déterminer un contenu rendu par l'énoncé

\section{Pierre croit que MS vient en T'}

qui, à son tour, est vrai si

$(\Sigma \mathrm{p})(($ Pierre croit $« \mathrm{p} ») \cdot($ Pierre croit « $\mathrm{p} » \equiv$ Pierre croit « MS vient en $\left.T^{\prime} »\right)$ ).

Il faut dire que cette dernière traduction n'est adéquate que si le verbe «croire » est entendu au sens matériel. Au sens intentionnel, il faudrait faire intervenir une traduction qui véhicule le même contenu informatif et qui correspond à la représentation que Pierre se fait de la situation. Notre énoncé initial pourrait alors être traduit en contexte par un énoncé comme «Pierre croit qu'il viendra demain », par exemple, qui doit se voir ensuite assigner une forme logique conforme à celle qui est indiquée plus haut pour les usages intentionnels.

\section{VI}

La traduction proposée pour l'énoncé «Pierre croit que Londres est jolie » est :

(1) $(\Sigma \mathrm{p})(($ Pierre croit $« \mathrm{p} ») \cdot($ Pierre croit $« \mathrm{p} » \equiv$ Pierre croit «Londres est jolie $))$.

Je fais ici toujours l'hypothèse que le verbe est utilisé matériellement. Lorsque le verbe est utilisé sur le mode intentionnel, la traduction adéquate du même énoncé est :

(2) ( $(\mathrm{q})(($ Pierre croit «Londres est jolie») (Pierre croit « q»). (Pierre croit « q» $\equiv$ Pierre croit « Pierre croit "Londres est jolie"»)).

En (2), toutes les occurrences du verbe « croire » illustrent un usage matériel, mais leur réitération permet d'exprimer l'usage intentionnel.

À quoi sommes-nous confrontés dans le scénario que nous présente Kripke? Ce scénario suppose que (1) soit vrai. Ensuite, 
étant donné que Pierre donne son assentiment à l'énoncé « London is not pretty ", on peut aussi conclure :

(3) $(\Sigma \mathrm{p})(($ Pierre believes $« \mathrm{p} ») \cdot($ Pierre believes $« \mathrm{p} » \equiv$ Pierre believes "London is not pretty") ).

En outre, on prétendra sans controverse que si Pierre donne un assentiment réflexif et sincère à un énoncé, il croit alors cet énoncé autant au sens intentionnel qu'an sens matériel. Cela veut dire qu'en plus des énoncés (1) et (3), l'énoncé (2) est vrai, ainsi que (4), qui est la version intentionnelle de (3):

(4) $(\Sigma q)$ ( (Pierre believes «London is not pretty ) · (Pierre believes " q") - (Pierre believes " q" $\equiv$ Pierre believes « Pierre believes "London is not pretty" »)).

Nous ne sommes pas capables encore de conclure que Pierre est confronté à une contradiction logique. Cependant, par le principe de traduction, il semble possible d'inférer (5) de (3):

(5) $(\Sigma \mathrm{p})(($ Pierre croit $" \mathrm{p} ») \cdot$ (Pierre croit $" \mathrm{p} » \equiv$ Pierre croit «Londres n'est pas jolie»)).

Cette dernière formule rapporte une croyance dont le contenu contredit celui qui est spécifié en (1).

On voudra peut-être s'objecter à cette inférence en invoquant le fait qu'elle constitue une application problématique du principe de traduction car, comme on l'a observé plus haut, il ne faudrait pas faire intervenir le principe pour attribuer à Pierre une relation à une phrase qu'il n'a pas vraiment utilisée. Dans de tels cas, en effet, les bonnes traductions ne se font pas salva veritate. Le principe ne devrait s'appliquer que pour les énoncés qui rapportent le contenu des croyances de l'agent. Toutefois, l'inscriptionnalisme suppose justement que le contenu d'une croyance s'épuise dans une phrase ou une inscription. On est donc en droit de faire intervenir le principe pourvu que l'on s'en tienne à des usages matériels des verbes psychologiques. Les véritables cas problématiques seraient ceux où l'on rapporte en style direct ce qu'un agent a dit.

Si un locuteur croit «London is not pretty » et si, comme nous le pensons, l'énoncé «Londres n'est pas jolie » en est une bonne traduction, il y a alors bel et bien un sens selon lequel il serait juste de dire qu'il croit en fait «Londres n'est pas jolie». En vertu du 
principe de traduction, (5) est vrai et cela implique que Pierre a des croyances contradictoires. Cette situation ne devrait pourtant pas nous inquiéter puisque les verbes en (1) et (5) n'ont qu'un usage matériel. La contradiction se produit en quelque sorte sans qu'il s'en rende compte.

Il serait toutefois gênant d'obtenir :

(6) $\left(\Sigma_{\mathrm{q}}\right)$ ((Pierre croit «Londres n'est pas jolie») - (Pierre croit « $»)$. (Pierre croit « q» $\equiv$ Pierre croit «Pierre croit "Londres n'est pas jolie"”)).

L'énoncé (6) semble pouvoir être inféré de (4) par une simple application du principe de traduction et il suppose une croyance dont le contenu contredit celui qui intervient dans la croyance rapportée en (2). Si l'on consent à utiliser le principe pour inférer (5) de (3), on ne voit pas pourquoi on s'objecterait à utiliser le principe pour inférer (6) de (4).

La situation est cependant fort différente. De (3) à (5), l'inférence est permise étant donné notre manuel de traduction. De (4) à (6), notre manuel est inutile et il faudrait plutôt avoir recours au manuel effectivement utilisé par Pierre. Or, Pierre opère avec un manuel de traduction dans lequel le mot «London » n'est pas traduit par le mot «Londres». Du moins est-ce là comment les choses apparaissent. Il faudrait plutôt dire que c'est exactement comme si Pierre avait un manuel de traduction dans lequel il n'y a pas de règle de traduction pour les mots «London » et "Londres ». Il en est ainsi parce qu'en réalité il n'utilise pas un manuel de traduction. Cela s'accorde parfaitement avec le fait qu'il soit un locuteur sémantiquement compétent. Nous n'avons en effet aucunement supposé que Pierre a traduit «London» par « Rome» et notre locuteur, rappelons-le, a acquis sa compétence de l'anglais en entrant directement en contact avec la population anglaise avoisinante. Nos traductions doivent quand même respecter le manuel que Pierre utiliserait ou qu'il serait disposé à adopter. Telle est du moins la contrainte à respecter pour la traduction des rapports intentionnels. L'énoncé (6) ne peut être inféré de (4) même si le principe de traduction s'applique toujours. La raison est que la «bonne traduction» doit apparaître dans le manuel de Pierre lorsqu'on lui attribue une croyance sur le mode intentionnel. La traduction d'un tel énoncé psychologique requiert que soient 
prises en considération les représentations que l'agent se fait de ses propres croyances. L'énoncé (6) affirme que Pierre se représente comme étant disposé à donner son assentiment à la phrase «Londres n'est pas jolie», mais nous savons tous très bien que Pierre ne se représente pas les choses de cette façon.

La traduction adéquate dans le cas d'un rapport intentionnel de croyance en est une qui recommande une traduction homophonique du contenu cité. Cette suggestion ne s'appuie pas sur une règle générale gouvernant la traduction des noms et suggérant que ceux-ci soient des expressions qui n'aient pas de traduction. Elle dépend plutôt du fait qu'il faut tenir compte, dans ce cas, de la représentation que l'agent se fait de ses propres croyances.

En somme, on peut proposer deux conventions pour la traduction des citations. La traduction anglaise d'une citation française doit contenir une citation d'une phrase anglaise sémantiquement équivalente lorsque la première est sous la portée d'une occurrence matérielle d'un verbe psychologique et une traduction homophonique lorsque le même verbe a une occurrence intentionnelle. Même si Pierre n'opère pas avec un manuel de traduction, les traductions homophoniques de ses contenus de croyance sont certes des traductions qui se conforment à la représentation qu'il se fait de la situation. La bonne traduction de (4) devrait alors être :

(7) $(\Sigma q)$ ( (Pierre croit «Londres n'est pas jolie») (Pierre croit "q»). (Pierre croit « q» $\equiv$ Pierre croit « Pierre croit "London is not pretty" »)).

qui ne fait plus intervenir un contenu de croyance intentionnelle qui contredise celui qui est attribué à Pierre en (2).

\section{VII}

Jusqu'à présent, notre solution semble reposer largement sur le fait que Pierre ne dispose pas nécessairement d'un manuel de traduction identique au nôtre et la question se pose de savoir si nous sommes en mesure de résoudre la version de l'énigme dans laquelle une seule langue est utilisée par Pierre. Selon Kripke, l'énigme peut réapparaître à l'intérieur d'une seule langue sans qu'il soit nécessaire de faire appel au principe de substitution des identiques et évidemment sans avoir à invoquer le principe de 
traduction. Le principe de décitation à lui seul suffit pour que l'on doive conclure, par exemple, que Pierre croit que Paderewski a un talent de musicien et n'a pas de talent de musicien. Il en est ainsi parce que Pierre ne sait pas que Paderewski, le politicien, est le même individu que Paderewski, le célèbre pianiste. Kripke insiste sur le fait que l'énigme surgit encore une fois ici parce qu'il veut montrer le caractère inadéquat de la solution qui consiste à stipuler que les noms propres n'ont pas de traduction admissible et que «Londres» est une expression qui doit être utilisée telle quelle dans toutes les langues. Pour rejeter cette solution, il fait intervenir cette nouvelle version de l'énigme dans laquelle Pierre n'est confronté qu'à des énoncés français ${ }^{12}$.

À vrai dire, si les énoncés auxquels Pierre donne son assentiment dans ce nouvel exemple sont interprétés comme ne contenant pas de déictiques, Pierre ne pourrait pas donner son assentiment aux deux car cela violerait la condition de réflexivité qui le rend apte à déceler les contradictions logiques dans les énoncés auxquels il voudrait souscrire. Pour que l'exemple s'accorde avec les hypothèses initiales, il faut que «Paderewski» fonctionne un peu comme une «expression directement référentielle » au sens de Kaplan ${ }^{13}$. Les expressions directement référentielles voient leur «sens » se ramifier en caractère et contenu. Le caractère est cette portion du sens qui rend l'expression sensible au contexte d'énonciation et se représente en sémantique formelle par une fonction de contexte dans contenu, alors que le contenu y est représenté par une fonction de monde possible dans dénotation. Ces «fonctions » représentent respectivement la signification linguistique et le contenu de l'expression. Dans le cadre de la sémantique proposée par Kaplan, les expressions indexicales sont des expressions directement référentielles dont le caractère est variable alors que les noms propres ont plutôt un caractère stable. En d'autres mots, les premières désignent un objet différent selon le contexte alors que les seconds désignent le même objet dans tous les contextes. Cette conception particulière de Kaplan concernant les noms propres ne peut toutefois pas être utilisée pour notre exemple car nous avons supposé que Pierre est

12. “A puzzle about belief », p. 265

13. D. KAPLAN, «On the Logic of Demonstratives» dans P. French et al. (eds), Contemporary Perspectives in the philosophy of language. U. of Minnesota Press, 1979. 
un locuteur sémantiquement compétent et il doit alors savoir que «Paderewski» est un nom propre.

Cela voudrait dire qu'il sait que le terme a un caractère stable et qu'il ne peut désigner un individu différent selon le contexte. Pour rendre l'exemple plausible, on doit donc amender la théorie de Kaplan et distinguer les noms propres des déictiques en stipulant que les premiers, contrairement aux seconds, peuvent avoir un caractère stable.

Une autre modification importante doit aussi être apportée à la théorie de Kaplan pour rendre l'exemple de Kripke plausible et conforme aux présuppositions initialement admises dans la formulation de l'énigme. Pour Kaplan, la compétence sémantique du locuteur implique la maîtrise de la «fonction-caractère » attachée à l'expression. Or, les noms propres, qui n'ont pas, selon Kaplan, de signification linguistique, voient leur caractère déterminé par la règle de dénotation dans laquelle un référent est assigné au nom. Si la compétence sémantique implique la maîtrise du caractère, Pierre devrait connaître les règles sémantiques associées aux noms propres de sa langue et ne pourrait pas entretenir des croyances contradictoires au sujet de Paderewski. Pour que l'exemple soit acceptable, il faut que les noms aient une signification linguistique à distinguer de la règle de dénotation. Par exemple, la signification linguistique de « $N$ » pourrait être celle qui est rendue par « L'individu nommé " $N$ " 》 et constituerait son caractère.

En bref, le terme «Paderewski», dans notre exemple, est une sorte d'expression directement référentielle dont le caractère peut être variable ou stable et que Pierre utilise de manière compétente sans toutefois maîtriser la règle de dénotation qui lui est associée. Puisque le nom a en fait un caractère stable, Pierre croit alors en fait au sujet d'une seule et même personne qu'elle est et n'est pas talentueuse sur le plan musical. Cela ne doit pas être considéré problématique puisque la croyance est dans ce cas-ci entendue au sens matériel. Sommes-nous cependant engagés à dire que Pierre se représente comme croyant des choses contradictoires au sujet d'une seule et même personne?

Conformément à la procédure d'élimination des termes déictiques indiquée dans la section $\mathrm{V}$, il faudrait déterminer le contenu de l'énoncé relativement au contexte, ce qui nous permet de faire 
porter la croyance de Pierre sur l'expression «Paderewski * ", une nouvelle expression dont le caractère est explicitement identifié comme étant stable. Pierre, en fait, croit que Paderewski* a et n'a pas un talent de musicien, mais il ne se représente pas comme quelqu'un qui croit que Paderewski * possède de cette manière une propriété et son complément. Pierre n'a pas des croyances intentionnelles contradictoires parce qu'il croit que le terme « Paderewski » a un caractère variable déterminé par l'existence présumée de deux règles sémantiques associées. La traduction intentionnelle de l'énoncé en contexte devrait être homophonique et relier Pierre à l'expression «Paderewski » elle-même.

Les distinctions que nous avons introduites gardent toute leur efficacité même dans ce nouvel exemple. Nous admettons que Pierre puisse avoir des croyances contradictoires, mais seulement au sens matériel du terme. Il n'a pas de croyances intentionnelles contradictoires et cela s'explique par le fait que les croyances intentionnelles s'analysent en des croyances matérielles que l'agent entretient au sujet de ses propres croyances. S'il se les représente comme étant dirigées vers des inscriptions, on est alors en mesure de comprendre pourquoi la perception qu'il a du caractère de l'expression est pertinente et détermine son attitude à l'égard de l'énoncé.

La distinction entre l'usage matériel et l'usage intentionnel, l'analyse de l'usage intentionnel en termes matériels et l'inscriptionnalisme permettent de voir comment l'énigme de Kripke peut être résolue. Dans tous les cas, il faut tenir compte de la représentation que Pierre se fait de la situation, ce qui revient à considérer le manuel de traduction avec lequel il opère ou encore la représentation qu'il se fait du caractère des expressions. De tels facteurs nous permettent de voir pourquoi Pierre ne se trouve pas intentionnellement en contradiction avec lui-même, ce qui, comme nous allons le voir, s'avère être une nouvelle plutôt réconfortante pour nous aussi.

\section{VIII}

J'ai respecté toutes les données de l'énigme de Kripke en la formulant sur le mode de dicto, en supposant que Pierre est sémantiquement compétent, réflexif, sincère, en admettant les 
principes de décitation et de traduction, sans pour autant être en mesure de dériver une contradiction chez Pierre. Pierre a sans doute des croyances contradictoires au sens matériel de "croire», mais nous ne sommes pas parvenus à des croyances intentionnelles contradictoires.

Nous allons maintenant voir que Pierre n'est pas plus dans la situation de ne pas croire que Londres est jolie, même en admettant la version forte du principe de décitation. Le fait que Pierre refuse d'accorder son assentiment à l'énoncé «London is pretty " nous justifie sans doute à conclure qu'il ne "croit» pas cette phrase, mais seulement au sens intentionnel du terme. Tout au plus, Pierre croit se trouver dans la situation d'un individu qui ne croit pas la phrase anglaise "London is pretty », mais nulle part avonsnous pu inférer la contradictoire de (1) ou (2). Plus précisément, si le verbe «croire » était dans ce cas utilisé sur le mode matériel, on pourrait appliquer le principe de traduction et obtenir alors la contradictoire de (1). Selon notre interprétation cependant, le refus d'accorder son assentiment implique seulement que l'agent ne la croit pas intentionnellement. Le principe de traduction doit s'appliquer en fonction du manuel de Pierre, ce qui nous empêche de faire valoir notre traduction et d'obtenir la contradictoire de (2).

Tout dépend de l'interprétation qui est accordée au principe de décitation dans sa version forte. Celui-ci stipule qu'un agent est disposé à donner son assentiment à une phrase " $\mathrm{p}$ » si et seulement si il croit que p. Le principe est certainement faux lorsque la croyance est entendue au sens matériel de l'expression. La notion d'assentiment introduite plus haut avec les contraintes de sincérité, réflexivité et compétence sémantique est bien évidemment une notion intentionnelle et il faudrait s'attendre à trouver aussi à droite de l'équivalence, dans la formulation du principe, une notion intentionnelle de croyance. Le principe serait, il est vrai, encore inacceptable s'il fallait admettre des croyances intentionnelles inconscientes mais il convient, pour les fins de la discussion, d'en rester aux états psychologiques conscients. Le principe peut alors être accepté tel quel. Nous avons vu, en vertu du principe faible de décitation, qu'un locuteur, qui donne son assentiment à une phrase, croit cette phrase autant au sens matériel qu'au sens intentionnel. On doit adopter cette interprétation parce que l'assentiment sincère est une condition suffisante pour la croyance 
intentionnelle, et cette dernière, une condition suffisante pour la croyance matérielle. Mais la croyance ne peut être entendue au sens matériel lorsque le principe de décitation se voit accorder la forme d'une bi-conditionnelle puisque l'agent peut croire matériellement une phrase " $\mathrm{p}$ » sans être pour autant disposé à donner son assentiment à cette phrase. En vertu de ce nouveau principe, le refus d'accorder son assentiment à « $\mathrm{p}$ » implique seulement que l'agent n'a pas la croyance intentionnelle que p. Étant donné ma définition d'une croyance intentionnelle, cela signifie que $\simeq(A$ croit matériellement « $\mathrm{p}$ ». A croit matériellement que $A$ croit matériellement " $\mathrm{p}$ ») ou, ce qui revient au même, que $\sim$ (A croit matériellement « $\mathrm{p} ») \mathrm{v} \sim(\mathrm{A}$ croit matériellement que $\mathrm{A}$ croit matériellement « $p$ »). Le refus d'accorder son assentiment à « $p$ » implique seulement que l'agent ne croit pas cette phrase ou qu'il ne croit pas qu'il la croit.

L'assentiment à une phrase n'implique pas que, de fait, l'agent ne croit pas sa contradictoire. De la même façon, un refus de se prononcer sur cette phrase n'implique pas que l'agent ne la croit pas matériellement. Ces situations doivent être reconnues si l'on est pour admettre la possibilité que l'agent puisse, de fait, croire des propositions contradictoires. Schématiquement, on doit reconnaître que

$$
\begin{gathered}
A \text { ass } 《 p » \rightarrow A \text { ne croit } p a s ~ « \sim p » \\
A \text { ref } « p » \rightarrow A \text { ne croit } p a s \text { « } p \text { » }
\end{gathered}
$$

lorsque la croyance est matérielle et lorsque «ass» et «ref» indiquent respectivement que l'agent accorde son assentiment à l'énoncé et qu'il refuse d'accorder cet assentiment.

Les inférences suivantes seraient toutefois permises:

$A$ ass " $p$ » $\rightarrow A$ croit " $A$ ne croit pas " $\sim p$ " » $A$ ref " $\mathrm{p}$ » $\rightarrow A$ croit "A ne croit pas " $\mathrm{p}$ " »

En admettant la version forte du principe de décitation, on est au pire engagé à dire que Pierre croit «Londres est jolie » et croit ne pas croire "Londres n'est pas jolie». En tant que locuteur de l'anglais, il croit "London is not pretty» et croit ne pas croire "London is pretty». Nulle part avons-nous que Pierre croit et ne croit pas la même phrase et, pour cette raison, aucune contradiction n'intervient chez celui qui rapporte les croyances de Pierre. 


\section{IX}

Il nous reste maintenant à examiner quelles conclusions peuvent être tirées des remarques précédentes concernant le fameux problème traditionnel de l'opacité référentielle. On pourrait penser que ce problème demeure, que les différents principes d'extensionalité échouent systématiquement en contexte épistémique de croyance et que, par conséquent, les solutions traditionnelles restent encore à envisager.

Il faut cependant reconnaître que le diagnostic habituel ne vaut plus lorsque l'on choisit d'adopter la solution que nous avons proposée. On ne peut plus prétendre que les contextes épistémiques soient sans exception référentiellement opaques. Notre solution, en effet, a des répercussions immédiates sur les discussions traditionnelles car les contextes épistémiques s'avèrent être référentiellement transparents lorsque les verbes sont utilisés sur le mode matériel. Rien ne s'oppose à ce qu'une croyance au sujet de Hesperus soit considérée équivalente à une croyance au sujet de Phosphorus, la substitution des identiques s'effectuant désormais salva veritate.

À première vue, il semble que la situation soit très différente lorsque le verbe est employé sur le mode intentionnel puisque la substitution des identiques ne s'y fait plus salva veritate. L'agent qui se représente comme quelqu'un qui croit que Hesperus est une planète ne se voit pas nécessairement comme quelqu'un qui croit la même chose au sujet de Phosphorus. Ce serait toutefois une erreur de penser que les contextes intentionnels de croyance, à savoir les contextes de la forme 'A BEL A BEL...', sont "par essence » référentiellement opaques. Le principe de substitution des identiques y est toujours applicable, sauf qu'il l'est à certaines conditions. Il faut faire intervenir des prémisses additionnelles qui concernent le savoir de l'agent. Dans notre exemple, il faudrait, pour que la substitution soit autorisée, que l'agent sache que Hesperus $=$ Phosphorus. Le principe s'applique donc toujours, mais à l'intérieur de contraintes imposées par les valeurs cognitives que l'agent attache aux expressions qu'il utilise. Il est vrai que la substitution ne peut être autorisée aussi longtemps qu'on ne dispose pas d'informations additionnelles suffisantes sur le savoir de l'agent auquel on attribue la croyance. Pour cette raison, les 
contextes épistémiques ont toutes les apparences d'être référentiellement opaques lorsque le verbe «croire» est utilisé sur le mode intentionnel, mais on ne peut parler au mieux ici que d'une opacité relative.

Il n'y a en réalité aucune différence de nature entre les rapports matériel et intentionnel, car le principe de substitution a aussi une application conditionnelle dans les contextes épistémiques matériels de croyance, les contextes de la forme 'A BEL...' dans lesquels n'apparaissent pas d'énoncés psychologiques au sujet de A. Les contraintes sont cette fois-ci imposées par les limites cognitives du rapporteur et le principe ne saurait être appliqué sans exception au fond que pour un rapporteur omniscient. Les contraintes sont toujours d'ordre cognitif, sauf qu'elles concernent les limites effectives du rapporteur et non plus celles de l'agent qui fait l'objet de l'attribution.

La situation peut être avantageusement comparée à celle observée plus haut concernant l'applicabilité du principe de traduction. Le principe devrait s'appliquer, avons-nous dit, en fonction du manuel utilisé par le rapporteur lorsque le verbe «croire» reçoit un usage matériel alors que seul le manuel de traduction de l'agent est utile lorsque le verbe est interprété au sens intentionnel. Cette situation ressemble un peu à celle qui nous occupe, le principe de substitution ne trouvant application qu'à l'intérieur des contraintes cognitives effectivement imposées par le rapporteur (pour un usage matériel du verbe «croire») ou par le tiers au sujet duquel se fait l'attribution (pour un usage intentionnel).

Comme chacun sait, Frege prend au sérieux l'échec du principe de substitution des identiques et l'explique en contexte épistémique par le fait que les termes y désignent leur sens ordinaire. Rien de tel ne doit être invoqué dans la perspective qui est la nôtre et la substitution des identiques peut se faire en contexte intentionnel pourvu que l'agent sache que Hesperus est Phosphorus. Notre optique se compare sur ce point à celle de Hintikka.

Contrairement à Hintikka cependant, il ne faut pas prétendre que la compétence sémantique de l'agent est fonction du savoir qu'il a de l'objet auquel il se réfère ou que sa référence est "multiple» aussi longtemps qu'il ne sait pas qui est ou quel est l'objet de sa référence. Il ne s'agit pas ici de prétendre que les 
valeurs cognitives que l'agent associe aux expressions ont une pertinence sémantique et qu'elles deviennent leurs véritables valeurs sémantiques lorsqu'elles apparaissent en contexte intentionnel. Dans un cadre inscriptionnaliste, le véritable objet d'une attitude psychologique de croyance est une inscription, rien d'autre, et il ne s'agit pas de faire intervenir au niveau sémantique les valeurs cognitives que l'agent attache à ces inscriptions. De la même manière qu'on a dissocié la compétence sémantique de Pierre de sa possession d'un manuel de traduction, il faut aussi séparer la compétence sémantique d'un agent à utiliser des expressions référentielles et le savoir que l'agent a de l'objet auquel il se réfère. L'agent peut être jugé sémantiquement compétent dès qu'il se réfère correctement à un objet par le biais d'une expression qui désigne effectivement l'objet, et ce, même s'il ignore l'existence d'expressions équivalentes qui désignent le même objet.

L'objet d'une attitude propositionnelle de croyance est une inscription et le fait que la substitution des identiques soit parfois interdite s'explique par le fait que l'agent se réfère à l'objet sous un certain aspect linguistique et qu'il a tendance à associer une valeur cognitive distincte à des expressions distinctes. L'échec de la substitution provient de ces différences dans les valeurs cognitives associées et l'erreur est seulement de penser que c'est là un phénomène qui doit, d'une manière générale, être jugé sémantiquement pertinent.

Département de philosophie, Université du Québec à Montréal 